\title{
Analysis of dominance for competitive ability in Drosophila melanogaster
}

\author{
J. R. de Miranda and \\ Paul Eggleston
}

Department of Genetics and Microbiology, University of Liverpool, Liverpool L69 3BX, U.K.

In these experiments the genetic basis of larval competition in Drosophila melanogaster was investigated. Competitive ability was defined by a series of regression coefficients relating larval performance to their mono- and duo-culture densities. Sixteen inter-related $F_{1}$ hybrids were individually compared with their parents, revealing the presence of large amounts of dominance and heterosis for the various competitive parameters, all directed towards improved competitive ability. Analysis of the $F_{1}$ hybrids amongst themselves revealed that most of the heterosis was due to either interchromosomal interaction, or the complementing action of haploid autosomes and relatively little was due to any specific interaction between the homologues. The relevance of these results to the current understanding of heterosis is discussed.

\section{INTRODUCTION}

A frequent observation in competition experiments is that the performance of an $F_{1}$ hybrid is generally superior to that of both parents (Robertson, 1960; Lerner and Ho, 1961; Dawson, 1966), an effect that even extends to crosses between different outbred populations (Vetukhiv, 1953; Brncic, 1954). The observed heterosis is probably due to the improved larval competitive ability of the $F_{1}$, although the general absence of larval density control in these experiments makes it difficult to quantify such effects. The development of yield-density regression analyses of competition has provided better control over the conditions for measuring competition, particularly the density and the amount or supply of food (Suehiro and Ogawa, 1980; Mather and Caligari, 1981; Wright, 1981; Spitters, 1983; Watkinson, 1984). The competitive influence of one individual on the performance of another individual (of the same or different genotype) is represented by the regression coefficients relating the average performance per individual to the mono- and duoculture densities of the various competitors. Four types of coefficient are produced. These are the absolute performance at a standard reference density ( $e$-value); the effects of monoculture density on performance (intra-genotypic competition); the influence of a genotype on the performance of other genotypes (inter-genotypic pressure) and the sensitivity of a genotype to competition from other genotypes (inter-genotypic sensitivity). Recent investigations into the genetic behaviour of these parameters (de Miranda and Eggleston, 1988c) and the related parameters aggression $(a)$ and response $(r)$ (Mather and Caligari, 1988; Hemmat and Eggleston, 1988) have revealed, besides the usual additive variation, high levels of heterosis for intergenotypic pressure and to a lesser extent for the $e$-values, as well as considerable amounts of dominance for inter-genotypic sensitivity. All dominance and heterosis was directed towards a competitively superior genotype and appeared to be primarily linked to chrornosomes II and III, with a slight emphasis on chromosome III (de Miranda, 1987; de Miranda and Eggleston, 1988c; Mather and Caligari, 1988). The precise role of the $\mathrm{X}$-chromosome is still unclear. Initial experiments have failed to locate any heterosis or dominance on the $\mathrm{X}$-chromosome for any of the parameters and only marginal levels of additive variation (de Miranda, 1987; Mather and Caligari, 1988). Although there is a good ecological reason for this (a strong competitive superiority for heterozygous females with respect to their hemizygous brothers would severely disrupt the sexratio), the data are, as yet, inconclusive. The pres- 


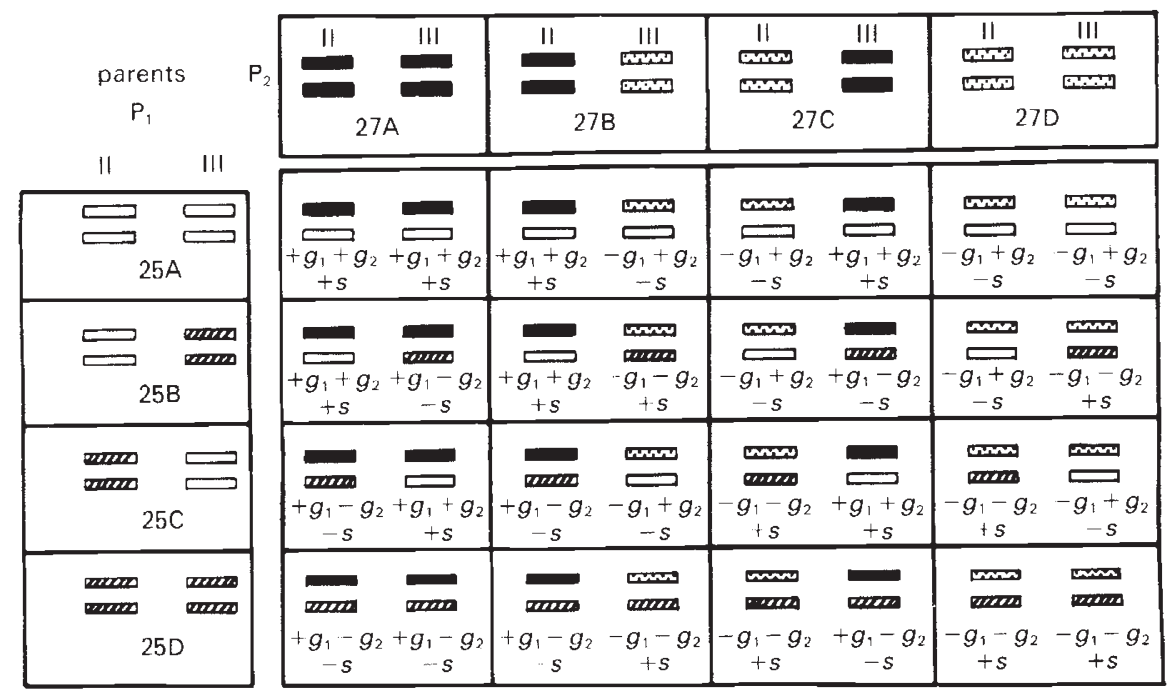

F, Hybrids

Figure 1 Schematic representation of the chromosomal constitution and the genetic expectations of the $16 \mathrm{~F}_{1}$ hybrids, obtained from intercrossing two different sets of substitution lines $(25 \mathrm{~A}, 25 \mathrm{~B}, 25 \mathrm{C}$, and 25D from a T19-T25 set of substitution lines and $27 \mathrm{~A}, 27 \mathrm{~B}, 27 \mathrm{C}$ and $27 \mathrm{D}$ from a T5-T27 set of substitution lines). In the analysis of the $F_{1}$ hybrids $g_{1}$ describes the difference between T5 (solid) and T27 (dotted) derived haploid chromosomes $\left(+g_{1}\right.$ for T5; $-g_{1}$ for T27), $g_{2}$ the difference between T19 (white) and T25 (striped) derived haploid chromosomes $\left(+g_{2}\right.$ for T19; $-g_{2}$ for T25) and $s$ the difference between a T5/T19 or T27/T25 combination $(+s)$ and a T5/T25 or a T19/T27 combination $(-s)$.

ence of large amounts of heterosis for competitive ability prompted the present investigation. A group of inter-related, heterozygous genotypes were compared amongst themselves, so as to describe the nature of the dominance in greater detail and to assess the extent of variation among genotypes which are representative of those found in the outbred parental population.

\section{MATERIALS AND METHODS}

Two sets of chromosome substitution lines were created as described by de Miranda and Eggleston (1988c), one between T19 and T25 and the other between T5 and T27, all of which are inbred lines of the Texas population (Linney et al. 1971). A selection of substitution lines from both sets were intercrossed as shown in fig. 1 , producing $16 \mathrm{~F}_{1}$ hybrids which vary for chromosomes II and III and are related by the ultimate origin of their chromosomes. The competitive profiles of the $16 \mathrm{~F}_{1}$ hybrids and the 8 parental lines were determined as described previously (de Miranda and Eggleston, 1987). Briefly, larval competition took place in $2.5 \mathrm{~cm} \times 7.5 \mathrm{~cm}$ vials containing $5.0 \mathrm{ml} 2$ per cent bactoagar as a non-nutritive base and $55.0 \mathrm{mg}$ live bakers yeast (YSC-2, Sigma) dispensed as a suspension. Each vial was seeded with the appropriate number of eggs of the various genotypes and all vials were incubated at $25^{\circ} \mathrm{C}$ until 10 days after the emergence of the first adults. The adult flies in each vial were collected daily and were separated according to body colour, counted and weighed to the nearest $0 \cdot 1 \mathrm{mg}$. For each of the $F_{1}$ hybrids and the parental lines one monoculture density series $(30,60,90$ and 120 eggs/vial) and one duoculture density series $[(30,90),(60,60)$ and $(90,30)$ eggs/vial $]$ were raised in duplicate, using a yellow bodied tester strain $\left(y^{2} \mathrm{~T} 25\right)$ as the second genotype in each duoculture series. The proportion of eggs surviving to adulthood $\left(p_{a}\right)$ and the mean weight of the adult flies in $\mathrm{mg}(\bar{w})$, both suitably transformed (de Miranda and Eggleston, 1987) were used as the two characters most likely to be affected by larval competition for food. The analysis of the density series is based on the assumption that an increase in density is synonymous with an increase in competitive stress, and that this can be linearly related to a change in the percentage survival and the mean adult weight. If we consider a primary, or indicator genotype $X$ and a secondary, or associate genotype $Y$ then the extent to which the larval survival is (adversely) affected by an increase in density is most easily represented by the slope of 
a regression of survival onto density $\left(c_{X X}\right)$; the greater the effect, the steeper the slope. A similar slope can be calculated if associate genotype eggs are added, instead of indicator genotype eggs. Considering only the indicator genotype, this slope represents the effect that each individual of the associate genotype has on the performance of the indicator genotype. The magnitude of this effect can be ascribed either to the competitive strength of the associate genotype (inter-genotypic pressure) or to the competitive weakness of the indicator genotype (inter-genotypic sensitivity). In addition to these slopes the regression analysis also estimates the $e$ value, representing the level of larval survival at the highest density $(120,0)$. Analysing the data for a genotype as indicator competitor produces estimates of its $e$-value, intra-genotypic competitive ability $\left(c_{X X}\right)$ and inter-genotypic sensitivity $\left(c_{X Y}\right)$, while analysing the data with the genotype as associate competitor yields the estimate of its inter-genotypic pressure $\left(c_{Y X}\right)$. Although both pressure and sensitivity describe a genotype's inter-genotypic competitive ability, and appear to be functionally related (de Miranda, 1987; Hemmat and Eggleston, 1988) empirical correlation coefficients between the two are rarely significant, and the parameters behave as if independently distributed. The experimental design and analysis used here are discussed in detail by Mather and Caligari (1981) and de Miranda and Eggleston (1987).

\section{RESULTS}

The performance of the $16 \mathrm{~F}_{1}$ hybrids and the parental lines are given in table 1 for each of the four competitive parameters (e-values, intragenotypic competition, pressure and sensitivity) and with respect to both competitive characters (larval survival and mean adult weight). The data for inter-genotypic pressure are also reproduced in fig. 2, as an illustration to aid interpretation. The significance of each dominance $(*)$ or heterosis (**) deviation of the $\mathrm{F}_{1}$ 's was determined at $P<$ 0.01 using $t$-tests. The most consistently heterotic

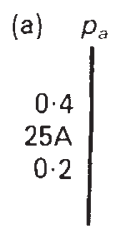

\begin{tabular}{r|}
0.4 \\
$25 B$ \\
0.2
\end{tabular}
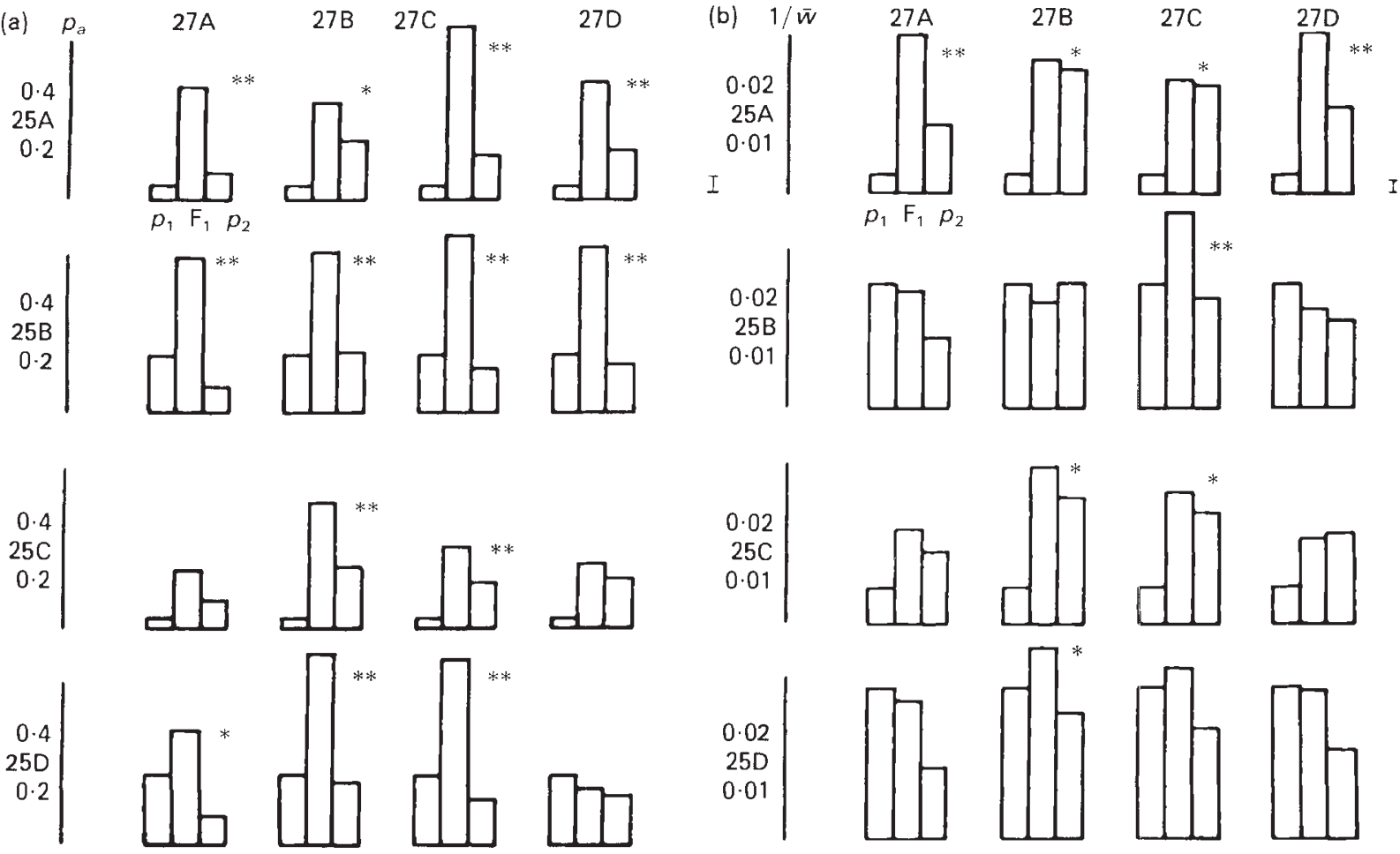

Figure 2 General pattern of dominance and heterosis for the $16 F_{1}$ hybrids when compared to their parents $p_{1}$ (rows) and $p_{2}$ (columns). Data are presented for inter-genotypic pressure only. Full results for all four competitive parameters are given in table 1. Fig. 2(a) relates to the proportion of eggs surviving to adulthood, converted to angles $\left(p_{a}\right)$ and fig. 2(b) to the mean adult weight $(\bar{w})$. A scale bar representing the standard deviation is included for each set of histograms. Significance levels for the dominance $\left(^{*}\right)$ or heterosis $\left(^{* *}\right)$ deviations were determined at $P<0 \cdot 01$ using $t$-tests. 
Table 1

(a) $e$-values

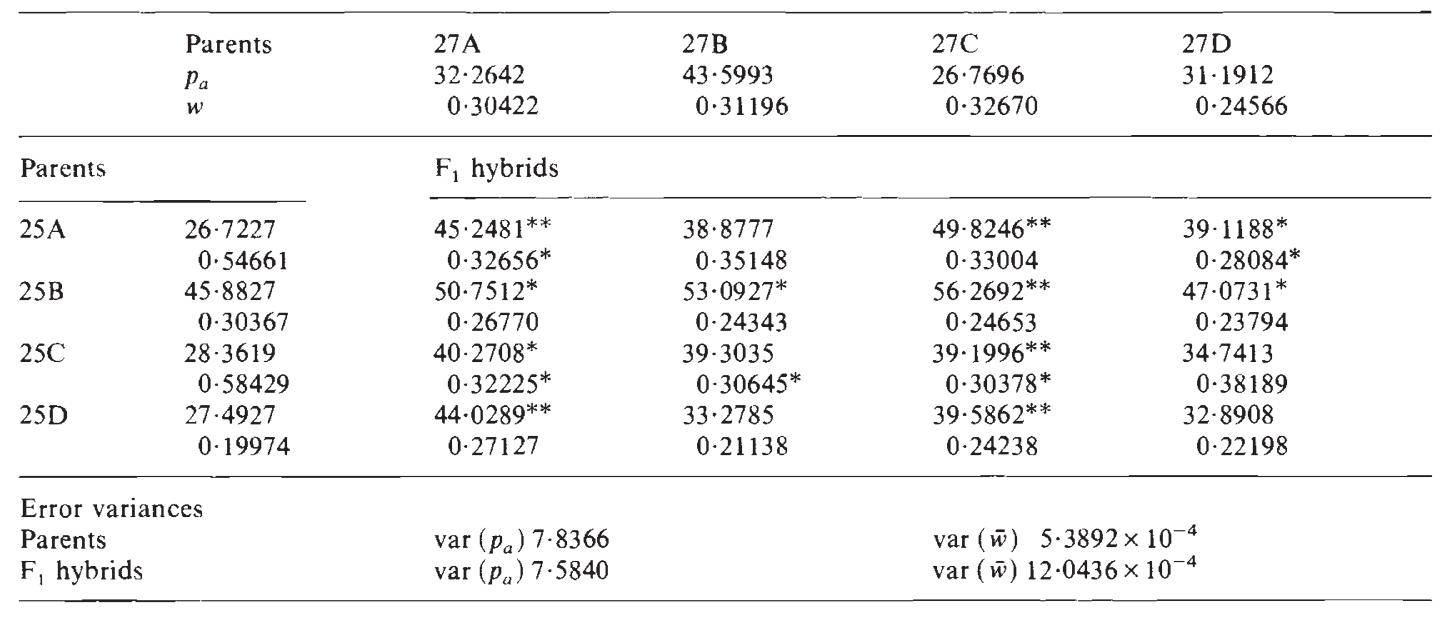

(b) Intra-genotypic competition $\left(c_{X X}\right)$

\begin{tabular}{|c|c|c|c|c|c|}
\hline & $\begin{array}{l}\text { Parents } \\
p_{a} \\
1 / \bar{w}\end{array}$ & $\begin{array}{l}27 \mathrm{~A} \\
0 \cdot 46157 \\
0 \cdot 016882\end{array}$ & $\begin{array}{l}27 \mathrm{~B} \\
0 \cdot 19918 \\
0.020091\end{array}$ & $\begin{array}{l}27 \mathrm{C} \\
0.57986 \\
0.016704\end{array}$ & $\begin{array}{l}27 \mathrm{D} \\
0.52160 \\
0.022958\end{array}$ \\
\hline \multicolumn{2}{|c|}{ Parents } & \multicolumn{4}{|c|}{$\mathrm{F}_{1}$ hybrids } \\
\hline \multirow[t]{2}{*}{$25 \mathrm{~A}$} & 0.00174 & $0 \cdot 16700$ & $0 \cdot 20839$ & $0 \cdot 13365$ & $0 \cdot 27567$ \\
\hline & 0.007261 & 0.020831 & 0.018277 & 0.020523 & 0.023546 \\
\hline \multirow[t]{2}{*}{$25 \mathrm{~B}$} & $0 \cdot 12414$ & $0 \cdot 13135$ & $0 \cdot 16764$ & 0.22326 & $0 \cdot 30793$ \\
\hline & 0.020794 & 0.027516 & $0.030842 * *$ & $0.030425^{* *}$ & 0.028831 \\
\hline \multirow[t]{2}{*}{$25 \mathrm{C}$} & 0.01636 & $0 \cdot 25209$ & $0 \cdot 23672$ & $0 \cdot 27147$ & $0 \cdot 10692$ \\
\hline & 0.008173 & 0.020784 & 0.022899 & $0.024910^{* *}$ & 0.017511 \\
\hline \multirow[t]{2}{*}{$25 \mathrm{D}$} & 0.52493 & 0.46559 & $0.55777^{*}$ & 0.45231 & 0.54249 \\
\hline & 0.034086 & 0.025425 & $0.037292^{*}$ & 0.031071 & 0.029480 \\
\hline \multicolumn{6}{|c|}{ Error variances } \\
\hline \multicolumn{2}{|c|}{ Parents } & \multicolumn{2}{|c|}{$\operatorname{var}\left(p_{a}\right) 2.7544 \times 10^{-3}$} & \multicolumn{2}{|c|}{$\operatorname{var}(1 / \bar{w}) 5.0468 \times 10^{-6}$} \\
\hline \multicolumn{2}{|c|}{$F_{1}$ hybrids } & \multicolumn{2}{|c|}{$\operatorname{var}\left(p_{a}\right) 2.7667 \times 10^{-3}$} & \multicolumn{2}{|c|}{$\operatorname{var}(1 / \bar{w}) 9.3246 \times 10^{-6}$} \\
\hline
\end{tabular}

(c) Inter-genotypic pressure $\left(c_{Y X}\right)$

\begin{tabular}{|c|c|c|c|c|c|}
\hline & $\begin{array}{l}\text { Parents } \\
p_{a} \\
1 / \vec{w}\end{array}$ & $\begin{array}{l}27 \mathrm{~A} \\
0.10213 \\
0.013267\end{array}$ & $\begin{array}{l}27 \mathrm{~B} \\
0 \cdot 22348 \\
0.023432\end{array}$ & $\begin{array}{l}27 \mathrm{C} \\
0 \cdot 16604 \\
0 \cdot 020657\end{array}$ & $\begin{array}{l}27 \mathrm{D} \\
0 \cdot 18520 \\
0 \cdot 016581\end{array}$ \\
\hline \multicolumn{2}{|c|}{ Parents } & \multicolumn{4}{|l|}{$F_{1}$ hybrids } \\
\hline \multirow[t]{2}{*}{$25 \mathrm{~A}$} & 0.04794 & $0.43374^{* *}$ & $0 \cdot 36855^{*}$ & $0.65202^{* *}$ & $0.44062 * *$ \\
\hline & 0.003752 & $0.029895^{* *}$ & $0.025423^{*}$ & $0.021483^{*}$ & $0.030096^{* *}$ \\
\hline \multirow[t]{2}{*}{$25 B$} & $0 \cdot 21661$ & $0.57996^{* *}$ & $0.60324^{* *}$ & $0.66709^{* *}$ & $0.61979^{* *}$ \\
\hline & $0 \cdot 023464$ & 0.023587 & 0.019783 & $0.036657^{* *}$ & 0.018587 \\
\hline \multirow[t]{2}{*}{$25 \mathrm{C}$} & 0.02927 & $0 \cdot 21304$ & $0.46498 * *$ & $0 \cdot 29963^{* *}$ & $0 \cdot 23831$ \\
\hline & 0.006393 & 0.017329 & $0.028881^{*}$ & $0.024229^{*}$ & 0.015536 \\
\hline \multirow[t]{2}{*}{$25 \mathrm{D}$} & $0 \cdot 24937$ & $0.41979^{*}$ & $0.69825^{* *}$ & $0 \cdot 68607^{* *}$ & $0 \cdot 20887$ \\
\hline & 0.027966 & 0.025363 & $0.035497^{*}$ & $0 \cdot 031714$ & 0.027333 \\
\hline \multicolumn{6}{|c|}{ Error variances } \\
\hline \multicolumn{2}{|c|}{ Parents } & \multicolumn{2}{|c|}{$\operatorname{var}\left(p_{a}\right) 2.4488 \times 10^{-3}$} & \multicolumn{2}{|c|}{$\operatorname{var}(1 / \bar{w}) 5.9776 \times 10^{-6}$} \\
\hline \multicolumn{2}{|c|}{$F_{1}$ hybrids } & \multicolumn{2}{|c|}{$\operatorname{var}\left(p_{a}\right) 6.1530 \times 10^{-3}$} & \multicolumn{2}{|c|}{$\operatorname{var}(1 / \bar{w}) 6.9893 \times 10^{-6}$} \\
\hline
\end{tabular}


Table 1 continued

(d) Inter-genotypic sensitivity $\left(c_{X Y}\right)$

\begin{tabular}{|c|c|c|c|c|c|}
\hline & $\begin{array}{l}\text { Parents } \\
p_{a} \\
1 / \bar{w}\end{array}$ & $\begin{array}{l}27 \mathrm{~A} \\
0 \cdot 61650 \\
0.021861\end{array}$ & $\begin{array}{l}27 \mathrm{~B} \\
0 \cdot 18421 \\
0 \cdot 018611\end{array}$ & $\begin{array}{l}27 \mathrm{C} \\
0 \cdot 62391 \\
0 \cdot 011504\end{array}$ & $\begin{array}{l}27 \mathrm{D} \\
0 \cdot 49783 \\
0 \cdot 017762\end{array}$ \\
\hline \multicolumn{2}{|c|}{ Parents } & \multicolumn{4}{|c|}{$F_{1}$ hybrids } \\
\hline \multirow[t]{2}{*}{$25 \mathrm{~A}$} & $0 \cdot 15638$ & $0 \cdot 10041^{*}$ & $0 \cdot 16027$ & $0.05031^{*}$ & $0 \cdot 24830$ \\
\hline & 0.023939 & 0.018038 & 0.017540 & 0.018917 & 0.024644 \\
\hline \multirow[t]{2}{*}{$25 \mathrm{~B}$} & $0 \cdot 13802$ & $0 \cdot 10425^{*}$ & 0.25419 & $0.15346^{*}$ & $0 \cdot 19693$ \\
\hline & 0.024994 & 0.019601 & 0.016096 & 0.016912 & 0.015225 \\
\hline \multirow[t]{2}{*}{$25 \mathrm{C}$} & 0.03206 & $0 \cdot 33167$ & $0 \cdot 13367$ & $0 \cdot 25134$ & $0.06285^{*}$ \\
\hline & 0.017254 & 0.015796 & 0.017583 & 0.016157 & 0.017686 \\
\hline \multirow[t]{2}{*}{$25 \mathrm{D}$} & 0.46785 & 0.44410 & 0.35209 & $0.22941^{* *}$ & $0.68787^{* *}$ \\
\hline & 0.026589 & 0.021685 & 0.019079 & 0.015163 & 0.020825 \\
\hline
\end{tabular}

Error variances

Parents

$F_{1}$ hybrids

$\operatorname{var}\left(p_{a}\right) 2 \cdot 3101 \times 10^{-3}$

$\operatorname{var}\left(p_{a}\right) 2 \cdot 292 \times 10^{-3}$

$\operatorname{var}(1 / \bar{w}) 4 \cdot 2328 \times 10^{-6}$

$\operatorname{var}(1 / \bar{w}) 7.8207 \times 10^{-6}$

Summary of the data generated by the experiment. Table 1(a) refers to the $e$-values in $p_{a}$ (top) and $\bar{w}$ (bottom) of the 8 parental lines and of the $16 \mathrm{~F}_{1}$ hybrids derived from them. The pooled error variance for the parental lines (56 df) and for the $F_{1}$ hybrids (112 df) are also given. Table $1(\mathrm{~b})$ similarly refers to estimates of intra-genotypic competition $\left(c_{X X}\right)$ in $p_{a}$ and $1 / \bar{w}$, with pooled error variances for $56 \mathrm{df}$ (parents) and $112 \mathrm{df}$ ( $\mathrm{F}_{1}$ hybrids). Table $1(\mathrm{c})$ refers to estimates of inter-genotypic pressure $\left(c_{Y X}\right)$ in $p_{a}$ and $1 / \bar{w}$, with error variances for $28 \mathrm{df}$ (parents) and $52 \mathrm{df}\left(\mathrm{F}_{1}\right.$ hybrids). Table $1(\mathrm{~d})$ refers to estimates of inter-genotypic sensitivity $\left(c_{X Y}\right)$ in $p_{a}$ and $1 / \bar{w}$, with pooled error variances for $56 \mathrm{df}$ (parents) and $112 \mathrm{df}\left(\mathrm{F}_{1}\right.$ hybrids).

For each of the $F_{1}$ hybrids an indication is given whether it deviates significantly from the mid-parental mean $(*$ dominance) and, where applicable, whether it also significantly exceeds the performance of the nearest parent $\left({ }^{* *}=\right.$ heterosis). Significance was determined at $P<0.01$ using $t$-tests. See also fig. 2 .

character was inter-genotypic pressure (fig. 2 and table 1(c)) both for survival and mean adult weight. Dominance, whether significant or not, was almost universally towards high inter-genotypic pressure. Since high pressure indicates competitive strength (Mather and Caligari, 1983) this suggests that the $F_{1}$ hybrids as a whole are better competitors than their parents. For inter-genotypic sensitivity (table 1(d)) the dominance levels are more intermediate, and generally towards reduced sensitivity, which is again a sign of competitive strength. The $e$-values (table 1(a)) also show considerable levels of dominance, positive for survival (extending occasionally into heterosis) and negative for the mean adult weight. The $e$-values concern the absolute performance at the highest density and consequently a large $e$-value for survival is an indication of competitive strength. The $e$-value for the mean adult weight is thought to be related to the minimum larval pupation weight (de Miranda and Eggleston, 1988c) and a low minimum pupation weight can be interpreted as a competitive asset (Bakker, 1961). Estimates for intra-genotypic competition tend to deviate in the opposite direction to the $e$-values (de Miranda, 1987) and this is by and large reflected in table 1(b). These findings concur with previous reports for such competitive parameters (de Miranda and Eggleston, 1988c) and absolute performance in a limiting environment (Robertson, 1961; Sang, 1964). Further inspection of the data in table 1 reveals that for all parameters investigated, even those displaying considerable levels of heterosis, there is still some overlap between the parental and the $F_{1}$ hybrid performances. It may therefore be possible to select for a homozygous line whose performance matches that of the $F_{1}$ hybrids shown here, especially since there is evidence for gene dispersion among these inbred lines (de Miranda and Eggleston, 1988c) which would account for at least some of the heterosis. Table 2(a) documents the correlations between the $F_{1}$ hybrid performances and their mid-parental means. These correlations reflect the heritability of the character, although this value may be inflated depending on the proportion of homozygous loci in the $F_{1}$ 's (where the $F_{1}$ is identical to both parents). The correlation coefficients in table 2(b) describe the relationship between the estimates of intergenotypic pressure and sensitivity. As was the case in previous reports (Mather and Caligari, 1983; Eggleston, 1985; de Miranda and Eggleston, 1987) 
Table 2 (a) Estimates of the correlation between the midparental mean and $\mathrm{F}_{1}$ hybrid performance for the $e$-values (e), intra-genotypic competition $\left(c_{X X}\right)$, inter-genotypic pressure $\left(c_{Y X}\right)$ and inter-genotypic sensitivity $\left(c_{X Y}\right)$. (b) Estimates of the correlation between inter-genotypic press(a) ure and inter-genotypic sensitivity

\begin{tabular}{lllll}
\hline$r_{14}$ & $e$ & $c_{X X}$ & $c_{Y X}$ & $c_{X Y}$ \\
$\begin{array}{l}\text { Larval } \\
\text { survival }\end{array}$ & 0.470 & $0.715^{* * *}$ & $0.533^{*}$ & 0.412 \\
$\begin{array}{l}\text { Mean adult } \\
\text { weight }\end{array}$ & $0.846^{* * *}$ & $0.784^{* * *}$ & 0.369 & $0.691^{* * *}$ \\
\hline
\end{tabular}

(b)

\begin{tabular}{lc}
\hline$r_{14}$ & $r\left(c_{Y X} ; c_{X Y}\right)$ \\
Larval survival & -0.344 \\
Mean adult weight & 0.253
\end{tabular}

All correlations are for 14 degrees of freedom. Significance levels are given as * $(0.05>P>0.01) ; * *(0.01>P>0.001)$; *** $(P<0.001)$.

there was no significant correlation between these estimates, supporting the contention that they are, at least in part, independent of each other (de Miranda and Eggleston, $1988 b ; 1988 c$; Hemmat and Eggleston, 1988). The $16 F_{1}$ hybrids can also be analysed amongst themselves by separating the general contributions of each haploid autosome $(g)$ and the specific interaction between pairs of homologues $(s)$ as shown in fig. 1 (the sex chromosomes are constant for all crosses). The recurrence of particular chromosome combinations means that there is a surplus of degrees of freedom after fitting the parameters. These can be used to test the significance of the residual variation, representing the interchromosomal interactions. The $g$ and $s$ parameters were estimated using a least squares analysis, and the residual variation was estimated as

$$
\chi_{(9)}^{2}=\sum_{i=1}^{16}\left(O_{i}-E_{i}\right)^{2} / s^{2}
$$

where $O_{i}$ and $E_{i}$ refer to the observed and expected estimates for each genotype, and $s^{2}$ refers to the average error variance of the $F_{1}$ hybrids. Examination of fig. 1 indicates that a positive value for $g_{1}$ represents a larger parameter estimate for the T5 derived chromosome than for the T27 derived chromosome. A similar argument applies to $g_{2}$ when considering the T19 and T25 derived chromosomes. Thus, for those competitive parameters where a large value is indicative of competitive strength (inter-genotypic pressure and the $e$-value for larval survival) a positive $g_{1}$ and $g_{2}$ represent the competitive superiority of the T5 and T19 chromosomes over the T27 and T25 chromosomes respectively. Conversely, for those parameters where a low value represents competitive strength (inter-genotypic sensitivity and the $e$-value for the mean adult weight) a negative $g_{1}$ and $g_{2}$ represent competitive superiority of T5 and T19 over T27 and T25 respectively. Estimates of the total variation $\left(\chi_{(15)}^{2}\right)$, the $g$ and $s$ deviations and the residual variation $\left(\chi_{(9)}^{2}\right)$ are shown in table 3 . The T19 chromosome-II and the T27 chromosome-II are competitively superior to the T25 and T5 chromosomes respectively, although only the T19 effects are significant. The T19 chromosomeIII is competitively inferior to the T25 chromosome with respect to the e-values and inter-genotypic pressure, but superior with respect to intergenotypic sensitivity. The T5 chromosome-III is superior for most parameters to the T27 chromosome, although this is significant in only one case ( $e$-value for larval survival). From these results it would appear that competitively desirable chromosomes, when considering their average effect in a range of heterozygous backgrounds, are dispersed throughout the inbred lines investigated here. Furthermore, the competitive parameters are (at least in part) under independent genetic control, since a chromosome may be simultaneously advantageous and disadvantageous, depending on which competitive parameter is investigated. These findings are in general agreement with previous reports (de Miranda and Eggleston, 1988c; Hemmat and Eggleston, 1988; Mather and Caligari, 1988). Of special interest are the $s$ interactions. These represent differences between the various homologue combinations and are therefore concerned with how the chromosomes interact to produce the $F_{1}$ phenotype. Since they are generally not significant, unlike the $g$ effects, the inference is that haploid homologues simply complement each other at those loci where their alleles differ, with little or no specific interaction between the alleles. Moreover, part of the $s$ effect could be due to variation in the amount of genetic overlap between different homologue combinations. For example, if T27 shares more loci with T19 than with T25, or if T29 carries more increasing alleles than T25, then the linear contribution of T27 is greater in a T27/T25 combination than in a T27/T19 combination (assuming unidirectional dominance at all loci), which can lead to an $s$ interaction. Despite the contribution of the individual chromosomes and the homologue interactions much of the variation between the hybrids remains unexplained (table 3 ). This residual variation, represented by $\chi_{(9)}^{2}$, is ascribed to interchromosomal interactions, primarily between 
Table 3

(a) Larval survival

\begin{tabular}{|c|c|c|c|c|}
\hline & $e$ & $c_{X X}\left(\times 10^{2}\right)$ & $c_{Y X}\left(\times 10^{2}\right)$ & $c_{X Y}\left(\times 10^{2}\right)$ \\
\hline$m$ & $42 \cdot 722^{* * *}$ & $28 \cdot 126^{* * *}$ & $47 \cdot 462^{* * *}$ & $23 \cdot 507^{* * *}$ \\
\hline \multicolumn{5}{|l|}{ Chr II } \\
\hline$g_{1}$ & $0 \cdot 384$ & -0.795 & -0.193 & 0.001 \\
\hline$g_{2}$ & $4 \cdot 810^{* * *}$ & $-7 \cdot 940^{* * *}$ & $7 \cdot 101^{* * *}$ & $-7 \cdot 656^{* * *}$ \\
\hline$s$ & -0.924 & $-2 \cdot 532$ & $-4.733^{*}$ & $-0 \cdot 375$ \\
\hline \multicolumn{5}{|l|}{ Chr III } \\
\hline$g_{1}$ & $2 \cdot 925^{* * *}$ & $-1 \cdot 918$ & 1.930 & $-2 \cdot 695^{*}$ \\
\hline$g_{2}$ & $-1 \cdot 899^{* *}$ & $-7 \cdot 478^{* * *}$ & $-8 \cdot 576^{* * * *}$ & $-6 \cdot 772^{* * *}$ \\
\hline$s$ & $-0 \cdot 112$ & $1 \cdot 874$ & -0.855 & $4 \cdot 303^{* * *}$ \\
\hline s.e. & 0.688 & $1 \cdot 315$ & $1 \cdot 961$ & $1 \cdot 197$ \\
\hline$\chi_{(9)}^{2}$ & $22 \cdot 319^{*}$ & $38 \cdot 959^{* * *}$ & $34 \cdot 662^{* * *}$ & $81 \cdot 587^{* * *}$ \\
\hline$\chi_{(15)}^{2}$ & $97 \cdot 6111^{* * *}$ & $118 \cdot 788^{* * *}$ & $73 \cdot 891^{* * *}$ & $170 \cdot 897^{* * *}$ \\
\hline proportion & 0.771 & 0.672 & 0.531 & 0.523 \\
\hline
\end{tabular}

(b) Mean adult weight

\begin{tabular}{lcccr}
\hline & $e\left(\times 10^{2}\right)$ & $c_{X X}\left(\times 10^{3}\right)$ & $c_{Y X}\left(\times 10^{3}\right)$ & $c_{X Y}\left(\times 10^{3}\right)$ \\
\hline$m$ & $28.349^{* * *}$ & $25.632^{* * *}$ & $25.549^{* * *}$ & $18 \cdot 179^{* * *}$ \\
Chr II & & & & \\
$g_{1}$ & 0.407 & -0.153 & -0.155 & -0.013 \\
$g_{2}$ & 0.207 & -0.537 & 0.525 & 0.182 \\
$\quad s$ & 0.766 & 0.214 & -0.477 & -0.552 \\
Chr III & & & & \\
$g_{1}$ & 0.532 & -0.449 & 1.118 & -0.395 \\
$g_{2}$ & $4.192^{* * *}$ & $-4.476^{* * *}$ & -1.440 & 0.105 \\
$s$ & -1.007 & 1.053 & $-1.993^{*}$ & -0.662 \\
s.e. & 0.868 & 0.764 & 0.685 & 0.699 \\
\hline$\chi_{(9)}^{2}$ & 5.614 & 10.469 & $60.454^{* * *}$ & 10.749 \\
$\chi_{(15)}^{2}$ & $30.627^{* *}$ & $48.197^{* * *}$ & $75.079^{* * *}$ & 12.677 \\
proportion & 0.817 & 0.783 & 0.195 & 0.152 \\
explained & & & & \\
\hline
\end{tabular}

Analysis of the variation between the $16 \mathrm{~F}_{1}$ hybrids. $g_{1}, g_{2}$ and $s$ are as defined in fig. 1 , and refer to the differences between haploid homologues and their interaction. $\chi_{(15)}^{2}$ and $\chi_{(9)}^{2}$ refer to the variation between the $16 \mathrm{~F}_{1}$ hybrids before and after fitting the $g$ and $s$ parameters respectively, such that $\chi_{(9)}^{2}$ represents the interaction between chromosomes II and III. The proportion of variation explained by the $g$ and $s$ parameters is also given. The standard error (s.e.) associated with the $m, g$ and $s$ parameters is based on either 112 degrees of freedom $\left(e, c_{X X}, c_{X Y}\right)$ or 52 degrees of freedom $\left(c_{Y X}\right)$. The significances of the $g$ and $s$ parameters were determined using $t$-tests, and are given as * $(0.05>P>0.01)$; ** $(0.01>P>0.001)$; *** $(P<0.001) . e, c_{X X}, c_{Y X}$ and $c_{X Y}$ refer to the $e$-values, intra-genotypic competition, intergenotypic pressure and inter-genotypic sensitivity respectively.

chromosomes II and III. For inter-genotypic pressure and sensitivity, which describe a genotype's relative competitive ability, as much as 50 per cent (larval survival) and 80 per cent (mean adult weight) of the variation is due to interchromosomal interaction, as compared with only 25 per cent for the $e$-values and $c_{X X}$, which describe a genotype's response to environmental limitation. The reason for this distinction is not clear. However, epistasis is often implicated in the maintenance of genetic variation in populations where it can buffer the effects of selection, and as such it is interesting that interchromosomal interactions are especially prevalent for those parameters describing a genotype's inter-genotypic competitive ability. Finally, the proportion of variation that can be explained by the $g$ and $s$ parameters bears a striking resemblance to the midparental mean $-F_{1}$ 
hybrid correlations in table 2(a). Both are in a sense heritability estimates, describing the performance of the $F_{1}$ hybrid in relation to that of its parents or in terms of its own haploid chromosomes and inter-homologue interactions. Since the latter analysis essentially bypasses dominance by redefining it in terms of the complementing action of dispersed alleles, plus their interaction, the inference is that the bulk of non-heritable variation is due to the interactions between chromosomes, rather than due to dominance. Only for the $e$-value survival and the sensitivity $\left(C_{X Y}\right)$ adult weight is there a marked difference between the two estimates, and the latter can be disregarded since neither the residual nor the total variation between the $F_{1}$ hybrids is significant. In fact, this lack of variation between the $F_{1}$ hybrids will cast doubt over the correlation coefficient as well.

\section{DISCUSSION}

Heterosis is believed to be due to a combination of gene dispersion and unidirectional dominance at most loci. The former ensures that the increasing and decreasing alleles are distributed between the parents, reducing the net additive effect whereas the latter ensures that at those loci for which the parents differ only the better allele is expressed in the $F_{1}$ hybrid, which therefore carries more increasing loci than either parent. The extent of heterosis is therefore also dependent on the number of dissimilar loci between the parents, the importance of each locus and the difference between the alleles. Such comparisons are unique for each pair of parents and hence $F_{1}$ performances cannot be completely predicted from the parental performances. This model of heterosis does assume that there is no specific interaction between the two alleles at each locus. Such interaction constitutes another explanation of heterosis and would suggest that heterozygosity per se, irrespective of the effect of the individual alleles, is beneficial to the $F_{1}$ hybrid. Although this type of interaction was found in these experiments, as inter-homologue $s$ effects, they were minor compared to the main contributions of the autosomal homologues. The presence of considerable amounts of interchromosomal interaction means that the specific combining ability of the genome as a whole is still significant. If these $F_{1}$ hybrids can be regarded as putative genotypes of the Texas population then the extent of variation between the hybrids and the proportion of this that can be linked to individual chromosomes (about half) provide a ready target for natural selection to act upon. This raises the question of how this variation is maintained, especially when considering the high levels of density related larval mortality encountered in cage populations and, by implication, the strong directional selection pressure for improved larval competitive ability. Both interchromosomal epistasis and the variation in larval ages in (semi) natural populations may be able to shield the genetic variation from the effects of natural selection (de Miranda and Eggleston, 1988c). Most of the heterosis for competitive ability is due to the inter-genotypic pressure of the competitors, a character believed to be closely related to the larval feeding rate (de Miranda, 1987; de Miranda and Eggleston, 1988a; $1988 \mathrm{~b}$ ). This is supported by direct studies of the larval feeding rate which also display large amounts of heterosis and aggregate dominance (Sewell et al., 1975; Burnet et al., 1977). The intermediate dominance levels for inter-genotypic sensitivity, thought to be more related to the resource utilisation efficiency, implies that dominance is less prevalent for this biological parameter. The components that comprise the larval feeding rate are modelled primarily as a series of functions, as opposed to a parallel set of functions. Consequently the feeding rate is prone to severe disruption due to the fixation of semi-deleterious alleles in the parents, causing heterosis in the $F_{1}$ hybrid (Burnet et al., 1977). In this light the utilisation efficiency may involve a range of mutually complementing functions, such that the fixation of several deleterious alleles need not necessarily disrupt the overall efficiency of the organism.

Acknowledgements We are grateful for the financial support from the S.E.R.C. and the Lister Institute of Preventive Medicine.

\section{REFERENCES}

BAKKER, K. 1961. An analysis of factors which determine success in competition for food among larvae of Drosophila melanogaster. Arch. Neerl. de Zool, 14, 200-281.

BRNCIC, D. 1954. Heterosis and the integration of genotypes in geographic populations of Drosophila pseudoobscura. Genetics, 39, 77-88.

BURNET, B., SEWELL, D. AND BOS, M. 1977. Genetic analysis of larval feeding behaviour in Drosophila melanogaster. II. Growth relations and competition between selected lines. Gen. Res. Camb., 30, 149-161.

DAWSON, P.S. 1966. Developmental rate and competitive ability in Tribolium. Evolution, 20, 104-116.

EGGLESTON, P. 1985. Variation for aggression and response in the competitive interactions of Drosophila melanogaster. Heredity, 54, 43-51. 
HEMMAT, M. AND EGGleston, P. 1988. Competitive interactions in Drosophila melanogaster: recurrent selection for aggression and response. Heredity, 60, 129-137.

LERNER, I. M. AND HO, F. K. 1961. Genotype and competitive ability of Tribolium species. Am. Nat., 95, 329-343.

LINNEY, R., BARNES, B. W. AND KEARSEY, M. J. 1971. Variation for metrical characters in Drosophila populations. Heredity, 27, 163-174.

MATHER, K. AND CALIGARI, P. D. S. 1981. Competitive interactions in Drosophila melanogaster. II. Measurement of competition. Heredity, 46, 239-254.

MATHER, K. AND CAligari, P. D. S. 1983. Pressure and response in competitive interactions. Heredity, 51, 435-454.

MATHER, K. AND CALIGARI, P. D. S. 1988. Competitive interactions in Drosophila melanogaster. IV. Chromosome assay. Heredity, 60, 355-369.

De MIRANDA, J. R. 1987. Ph.D thesis, University of Liverpool, U.K.

DE MIRANDA, J. R. AND EGGLESTON, P. 1987. A comparison of substitution and addition designs for the analysis of competitive interactions in Drosophila melanogaster. Heredity, 58, 279-288.

DE MIRANDA, J. R. AND EGGLESTON, P. 1988a. Larval competition in Drosophila melanogaster. I. Estimation of larval growth parameters. Heredity, 60, 205-212.

DE MIRANDA, J. R. AND EGGLESTON, P. 1988 b. Larval competition in Drosophila melanogaster. 11. Comparing biological and competitive parameters. Heredity, 60, 213-219.

DE MIRANDA, J. R. AND EgGleston, P. 1988c. Genetic analysis of larval competition in Drosophila melanogaster. Heredity, 61, 339-346.
ROBERTSON, F. W. 1960. The ecological genetics of growth in Drosophila. 3. Growth and competitive ability of strains selected on different diets. Gen. Res. Camb., 1, 333-350.

ROBERTSON. F. W. 1961. The ecological genetics of growth in Drosophila. 4. The influence of larval nutrition on the manifestation of dominance. Gen. Res. Camb., 2, 346-360.

SANG, J. H. 1064. Nutritional requirements of inbred lines and crosses of Drosophila melanogaster. Gen. Res. Camb., 5, $50-67$.

SEWELL, D., BURNET, B. AND CONNOLLY, K. 1975. Genetic analysis of larval feeding behaviour in Drosophila melanogaster. Gen. Res. Camb., 24, 163-173.

SPITTERS. C. J. T. 1983. An alternative approach to the analysis of mixed cropping experiments. 1. Estimation of competition effects. Neth. J. Agric. Sci., 31, 1-11.

SUEHIRO, K. \& OGAWA, H. 1980. Competition between two annual herbs, Atriplex gmelini C. A. Mey and Chenopodium album L. in mixed cultures irrigated with seawater of various concentrations. Oecologia, 45, 167-177.

VETUKHIV, M. 1953. Viability of hybrids between local populations of Drosophila pseudoobscura. Proc. Nat. Acad. Sci., $39,30-34$.

WATKINSON, A. R. 1984. Yield-density relationships: the influence of resource availability on growth and self-thinning in populations of Vulpia fasciculata. Ann. Bot., 53, 967-976

WRIGHT, A. J. 1981. The analysis of yield-density relationships in binary mixtures using inverse polynomials. J. Agric. Sci. Camb., 96, 561-567. 